\title{
Lithium enhancement in X-ray binaries due to stellar rotation ${ }^{\star}$
}

\author{
T. J. Maccarone ${ }^{1}$, P. G. Jonker ${ }^{2,4}$, and A. I. Sills ${ }^{3}$ \\ 1 Astronomical Institute “Anton Pannekoek”, University of Amsterdam, Kruislaan 403, 1098 SJ Amsterdam, The Netherlands \\ e-mail: tjmascience.uva.nl \\ 2 Harvard-Smithsonian Center for Astrophysics, 60 Garden Street, Cambridge, MA 02139, USA \\ 3 Department of Physics and Astronomy, McMaster University, Hamilton, Ontario, L8S 4M1, Canada \\ ${ }^{4}$ SRON, National Institute for Space Research, 3584 CA, Utrecht, The Netherlands
}

Received 27 December 2004 / Accepted 9 March 2005

\begin{abstract}
We discuss the high lithium abundances in the secondary stars of X-ray binaries. We show that no lithium production in these stars is necessary, and that the abundances can be explained simply due to the tidally locked rotation of the stars, which lead naturally to slower lithium destruction rates. The differences in abundances of CVs' secondaries from those of LMXBs had previously been put forth as evidence that the compact object was related to the lithium abundance, but this scenario also accounts for the lower lithium abundances in the secondary stars in cataclysmic variable systems (CVs) than in low mass X-ray binaries (LMXBs), since these stars have typically lived much longer before becoming tidally locked short period systems. We point out that if this scenario is correct, then the globular cluster X-ray binaries' donor stars should, as a class, show less lithium enhancement relative to other stars of the same spectral type in the clusters than the field X-ray binaries donor stars show.
\end{abstract}

Key words. stars: abundances - binaries: close -X-rays: binaries

\section{Introduction}

Light element abundances (deuterium, helium and lithium) are a key tracer of the physical conditions in the early universe, as these are the only elements produced in the Big Bang. They provide a key constraint on the baryon-to-photon ratio at the time of the universe's formation. As a result, considerable effort has been invested in determining the rate at which lithium is destroyed, and in identifying the stars which have the highest lithium abundances. A key factor for understanding how to convert the observed lithium abundances observed in stars today into primordial values is to understand mechanisms for lithium production, rather than just lithium destruction. It has been noted in several cases that the lithium abundances in $\mathrm{X}$-ray binary donor stars are substantially larger than the solar value (Martin et al. 1994; Martin et al. 1996, and references within); this effect is seen in all X-ray binary secondary stars where there are good constraints on the lithium abundances. Since compact objects are natural sites for the acceleration of relativistic particles or flows, several mechanisms related directly to the compact object nature of the systems have been suggested - spallation in the inner accretion flow (Martin et al. 1995; Yi \& Narayan 1997), spallation due to the impact

\footnotetext{
* Chandra Fellow.
}

on the stellar atmosphere of high energy neutrons produced in the inner accretion flow (Guessom \& Kazanas 1999), or interactions between a relativistic jet and the mass donor (Butt et al. 2003), or alternatively, lithium production due to some unspecified process in stellar coronae (Bildsten \& Rutledge 2000). With the exception of the last two mechanisms, the transport of the lithium back into the stellar atmosphere is a non-trivial problem. In this paper, we will review the evidence that X-ray binaries' donor stars have higher than normal lithium abundances. We will then demonstrate the similarities with other tidally locked binary stars, and will discuss the possibility that this lithium enhancement may be a natural consequence of the slower lithium depletion in tidally locked binary stars, rather than a consequence of any physics related to the compact objects. We will explain, in the context of this picture, why this enhancement is not seen so strongly in cataclysmic variable stars $(\mathrm{CVs})$.

\section{Summary of past X-ray binary observations and normal star observations}

There are, at the present time, five secondary stars in X-ray binaries with measured lithium abundances. The measured abundances, expressed as, $A(\mathrm{Li})=\log N(\mathrm{Li}) / N(\mathrm{H})+12.0$, range 
from 2.2 to 3.3 , where the solar value is approximately 1.0 , so these stars are above the solar value by a factor of about 20-200. The actual enhancement relative to what is expected based on normal single star evolution depends heavily on the unknown stellar age and on the stellar mass (see e.g. the $\approx 2 \mathrm{dex}$ difference between the cool stars in the 80 Myr old Pleiades and in the 700 Myr old Hyades compiled by Ryan \& Deliyannis 1995). The lithium abundance values of X-ray binary donors are all presented in Martin et al. (1996), with the exception of that for Cen $\mathrm{X}-4$, which has $A(\mathrm{Li})=3.3$, and is reported separately (Martin et al. 1994). Martin et al. (1996) also note that the ionizing radiation from the X-ray source might lead to an underestimate of the lithium abundance.

The meteoritic lithium abundance in the solar system has a value of $3.31 \pm 0.04$ (Anders \& Gravesse 1989). This is generally accepted to be the typical initial lithium abundance of Population I stars (e.g. Ryan \& Deliyannis 1995). Hence, even the most lithium-rich of the X-ray binaries, Cen X-4, which has a lithium abundance equal to this value, does not require any lithium enhancement of the secondary star, so long as there has not been substantial lithium depletion in this star over its lifetime, and so long as the underestimate due to the lack of accounting for ionization effects is not too severe.

A wealth of literature, both observational and theoretical, is devoted to the effects of rotationally-induced mixing on lithium depletion in stellar atmospheres. It has been suggested that rotating stars should deplete their initial atmospheric lithium abundances more slowly than do non-rotating stars, and that this slower depletion of lithium should be an especially strong effect in tidally locked binaries where internal shears (which mix the lithium into the inner regions of the star where it is destroyed) will be prevented from developing due to the very slow angular momentum losses of the stars (Pinsonneault et al. 1990). Furthermore, it has been found in open clusters that the short-period (i.e. with periods less than 6 days) tidally locked binary systems have substantially higher lithium abundances than single stars of the same type, or stars of the same type in wide binaries; the most lithium-rich stars in open clusters are these short-period tidally locked binaries, and they often show lithium abundances rather close to the meteoritic value (see e.g. Soderblom et al. 1990; Ryan \& Deliyannis 1995). These results stand in contrast to the claim of Martin et al. (1994) that, while tidal locking of the LMXBs might play some role in their enhanced lithium abundances, it was likely to be a rather minor effect.

At the present time, only a small number of open cluster tidally-locked binaries have measured lithium abundances (9 appear in the paper of Ryan \& Deliyannis (1995), and there has not been any systematic study made since then). Seven of the nine systems have $A(\mathrm{Li})>1.8$ and four have $A(\mathrm{Li})$ of at least 3.0, indicating that the distributions of the X-ray binary systems and the open cluster systems are in relatively good agreement. However, the open cluster systems typically have hotter stars, so it is a better comparison to look at their overabundances relative to the mean trend with temperature for the clusters in which they are found; most of these systems are at least 0.6 dex higher in lithium than the trend curve in lithium versus effective temperature of the open clusters of which they are members. The two Pleiades systems show no measureable lithium enhancement, but this is a fact which is in agreement with theoretical models of rotationally induced lithium depletion given the youth of the Pleides cluster.

The data is not as conclusive for subgiant stars. For two subgiants in the Hyades, lithium is detected at temperatures where there are only upper limits for stars which are not short period tidally locked binaries and where the trend line extrapolates to be about 100 times lower than the detections. On the other hand, the lithium abundances are lower than $A(\mathrm{Li})=1.8$ in these two stars. Clearly, more observations of tidally-locked subgiants are needed to determine whether tidal locking can account for a substantial fraction of the lithium excess in X-ray binaries with subgiant donors.

A typical factor of $\sim 5$ enhancement in lithium abundance is thus found for the tidally locked binaries in open clusters. Furthermore, it should be noted also that the effects of tidal locking for the donor stars in X-ray binaries should extend much deeper into the stellar interior than for those systems compiled by Ryan \& Deliyannis (1995). The main sequence systems studied by Ryan \& Deliyannis (1995) all have orbital periods greater than 3 days, while all of the X-ray binaries except V404 Cyg in the sample have periods less than 1 day and most have periods less than $10 \mathrm{~h}$. Pinsonneault et al. (1989) studied the lithium depletion as a function of the timescale for angular momentum transport between the surface and the stellar interior; they found it to have "a strong influence" on lithium depletion, capable of accounting for 2 dex of variation over the range they studied. The model they ran for a solar-type star with weak angular momentum transport retained $A(\mathrm{Li})$ greater than 2.5 for about $300 \mathrm{Myr}$, and greater than 2.0 for approximately 5 Gyr. The donors in X-ray binaries should have essentially no angular momentum transport within the star; it therefore seems quite reasonable that they could have more lithium enhancement than in the case of the wider binaries.

\section{Discussion}

\subsection{Past interpretations}

One of the strongest arguments made in the past for the position that the enhancement of lithium in X-ray binary donor stars must be related to the compact object, rather than to the stellar rotation, is the point that the stellar companions in cataclysmic variables show no evidence for lithium enhancement (Martin et al. 1995; Yi \& Narayan 1997). This argument is flawed, though, if one assumes, as is predicted by stellar theory and borne out by stellar observations, that lithium is constantly being destroyed at a rather slow rate in short-period tidally locked binary systems, while it is being destroyed at a much faster rate in stars with low rotation rates. The black hole and neutron star binaries are the end products of evolution of very massive stars with very short lifetimes. The progenitors of the white dwarfs found in cataclysmic variable systems, on the other hand, have considerably longer lifetimes. Since the orbital periods of these systems shrink rather late in their evolution, it is likely for most CVs that while the white dwarf 
progenitor was on the main sequence, the binary was not tidally locked, so the lithium depletion was proceeding in more or less the normal fashion for isolated or wide binary stars. In fact, it is then likely that some of the cataclysmic variable's donor stars will show mildly enhanced lithium overabundances, but since a rather large amount of lithium depletion probably occurs in the first $100 \mathrm{Myr}$ of stellar evolution (see e.g. Pinsonneault et al. 1990), CVs' secondaries should be substantially more lithium depleted than neutron star or black hole binaries.

On the other hand, Bildsten \& Rutledge (2000) drew attention to the similarity between X-ray binaries' donor stars and the members of RsCvn systems. The stars in RsCvn systems are likely to be tidally locked short period binaries from rather early on in their evolution. Bildsten \& Rutledge (2000) had been arguing that the $\mathrm{X}$-ray emission from quiescent low mass $\mathrm{X}$-ray binaries was primarily from the stellar coronae of the mass donors, rather than from a low luminosity accretion flow. In that context, they argued that since both quiescent LMXBs and RsCvn stars had enhanced lithium abundances and X-ray emission (as does at least one white dwarf binary system), that perhaps both were caused by the coronal activity. On top of the arguments of Bildsten \& Rutledge (2000) that the lithium enhancement is unlikely to be caused by processes related directly to accretion onto compact objects, we note that the secondary with the highest lithium abundance, by far, is that in Cen X-4, the only neutron star X-ray binary whose secondary's lithium abundance has been measured. Many of the mechanisms for enhancing lithium abundance in X-ray binaries' secondary stars are related to phenomena which are considerably stronger in black hole systems than in neutron star systems - relativistic jets (the mechanism of Butt et al. 2003) from black holes are considerably more powerful than those from neutron stars (see e.g. Migliari et al. 2003), and the radiatively inefficient accretion flows (the mechanism of Yi \& Narayan 1997) seen from black hole transients are generally hotter than those seen from neutron star transients.

\subsection{Interpretation in terms of stellar rotation effects}

In fact, though, it is not necessary to produce lithium in X-ray binaries. We note that a large part of the reason why it is thought that the donor stars in X-ray binaries are enhanced in lithium is that they are far more lithium abundant than is the Sun. For most elements, substantial deviations from solar abundances are indicative of some unusual evolutionary history. However, the solar lithium abundance at birth was $A(\mathrm{Li})=3.3$, and over the 5 Gigayear lifetime of the Sun, this value has fallen by a factor of about 200. Therefore, if lithium depletion is halted in the very high rotation rate donor stars like the secondaries in X-ray binaries, then it would not be surprising to see these systems with lithium abundances closer to the standard value at formation for Population I stars, rather than at the solar or sub-solar values typically seen in evolved Population I stars. An exact quantification of this effect is well beyond the scope of this paper; calculations of lithium depletion in tidally locked binaries have not yet been done. The angular momenta of the secondary stars in most X-ray binaries are an order of magnitude higher than the fastest rotators studied by Pinsonneault et al. (1990), and furthermore, the lithium depletion is further stemmed due to the very slow angular momentum loss in these systems. Zahn (1994) attempted to estimate the lithium depletion as a function of angular momentum for tidally locked binaries, but extrapolating from the relation presented in that paper gives a lithium abundance well in excess of the meteoritic value. It thus seems most likely that the secondary stars in X-ray binaries undergo some weak lithium depletion early in their lifetimes, before the supernova explosion creating the compact object, and then undergo little or no further lithium depletion, but considerable further theoretical work should be done to test this hypothesis.

A possible problem with this mechanism for lithium enhancement is the mass loss from the stellar envelope during the system's accreting phase. We note, though, that the stars for which the Li 6708 Angstrom line can be observed are the cooler stars among the X-ray binary counterparts. As a result, these fall into two categories - subgiants with relatively long periods whose lithium abundance evolution is poorly understood both theoretically and observationally (a category including only V404 Cyg, whose orbital period is 6.5 days), and main sequence stars with quite short periods - all the other lithium rich secondaries are in systems with orbital periods shorter than 1 day (with most of them being shorter than $10 \mathrm{~h}$ ). It has been shown on both theoretical and observational grounds (Brocksopp et al. 2004; Meyer-Hofmeister 2004; Portegies Zwart et al. 2004) that the shortest period X-ray binaries have the lowest outburst amplitudes and the lowest mean mass accretion rates among X-ray binaries. The theoretical calculations of the expected mass accretion rates from systems in this orbital period range are of order $3 \times 10^{-10} M_{\odot} \mathrm{yr}^{-1}$ (King et al. 1996), so at least $1 \mathrm{Gyr}$ would be required before these systems underwent substantial lithium depletion due to stripping of the outer layers of the stars. Furthermore, while starting from orbital periods shorter than those of the open cluster systems which have been studied, LMXBs will typically require several Gigayears of evolution as tight, tidally locked binaries before actually coming into Roche lobe contact and starting accretion (e.g. Kalogera \& Webbink 1998), further prolonging their lifetimes as lithium rich objects. Many short period X-ray binaries should thus have pre-accretion lifetimes of at least a substantial fraction of a Hubble time, allowing most of them to remain as lithium rich objects today.

\subsubsection{Observational tests}

We can make one rather strong prediction based on the suggestion that LMXB secondary stars have enhanced lithium because of reduced depletion, and rather than because of actual lithium production. The mass donors in globular cluster X-ray binaries should, as a class, show less lithium enhancement when compared with other stars of similar spectral types in the same clusters than the mass donors in non-cluster X-ray binaries. The 
reason for this is that the X-ray binaries in globular clusters are formed through dynamical captures (Clark 1975) - either tidal interactions (Fabian et al. 1975) or three-body (or perhaps fourbody) exchange interactions (Hills 1976). As a result, many of these stars will have undergone normal stellar evolution for most of their lifetimes before they became part of a close binary. It should be noted that there is one millisecond pulsar in the globular cluster NGC 6397 whose binary companion shows a lithium enhancement (Sabbi et al. 2003), but it does not seem likely that good measurements of the lithium abundances of a large sample of globular cluster X-ray binary secondary stars will be made with current instrumentation; this task requires both a large aperture (i.e. greater than that of the HST) and very good angular resolution (i.e. better than that available from the ground without adaptive optics). Plans exist for allowing adaptive optics measurements in the red part of the optical spectrum in the near future, so there is some hope for executing this test of rotational lithium enhancement in X-ray binary secondaries in the near future.

Similarly, the stars in low mass X-ray binaries with rotation powered pulsars (i.e. high magnetic field neutron stars) as their compact objects should also be a good test bed for theories of lithium production; that these systems contain slowly spinning, high magnetic field neutron stars is probably indicative of a much shorter history as an accretor; furthermore, these systems are the one class of X-ray binaries that have never been seen to show relativistic jets (Fender 2005), and the highest energies in their X-ray spectra are much lower than those seen in the low/hard states of low magnetic field neutron stars or black holes.

If it can be proven that the lithium abundances in X-ray binaries' stellar counterparts are the result of preservation of lithium due to stellar rotation early in the stars' lifetimes, rather than some means of strong lithium production, this would provide us with a natural means for determining which neutron stars in binaries were formed in supernovae, and which were formed in accretion induced collapses. It has been suggested in some cases that accretion onto a white dwarf, in certain mass accretion rate regimes, can lead to the accumulation of enough mass to allow the white dwarf to exceed the Chandrasekhar limit, and to become a neutron star (e.g. Bailyn \& Grindlay 1990; van den Heuvel \& Bitzaraki 1995). Since these systems will have undergone a temporary phase as an accreting white dwarf, and hence they will have had mass donors which were not rapid rotators until relatively late in their lifetimes, they should have lithium abundances in their secondary stars which are similar to those of the cataclysmic variables (i.e. not significantly enhanced relative to field stars), rather than to those of the other low mass X-ray binaries. At the present time, it is rather difficult to test this hypothesis; the systems suggested to have evolved into neutron stars through accretion induced collapse are either globular cluster systems (Bailyn \& Grindlay 1990) or they are systems in which the donor stars themselves are white dwarfs (van den Heuvel \& Bitzaraki 1995). In the former case, the neutron star may have changed partners over its evolution, while in the latter cases, the expected lithium abundance is not clear. Furthermore, at the present time, the measurements of lithium abundances in the neutron star
LMXBs' secondaries are quite poor; there exists only the detection of lithium in Cen X-4 and an upper limit in Aql X-1 which is not sufficiently low as to be constraining (Garcia et al. 1999).

Another apparently natural test would seem to be a measurement of the ${ }^{6} \mathrm{Li} /{ }^{7} \mathrm{Li}$ ratio, but the lines are very close together, and the ${ }^{6} \mathrm{Li}$ abundance measurements are typically considerably lower than the ${ }^{7} \mathrm{Li}$ abundances. To make useful measurements would require a very long integration on a very large telescope, but would also require that the system to be observed is nearly face-on, so that the orbital and rotational broadening of the lines is minimized. A more feasible test would be to measure the Be abundances. If the lithium overabundances in X-ray binary secondaries are due to tidal locking preserving lithium, rather than lithium production, then one would expect the $\mathrm{Be}$ abundances to track the lithium abundances in the same way as they do in other tidally locked binaries. Because the strongest Be lines are at 3130 Angstroms, this would require the source to be nearly directly overhead in order to minimize the atmospheric absorption. Fortunately, this is possible with large planned or existing flexibly scheduled telescopes for two of the systems with strong lithium enhancements - V404 Cyg, which is sometimes nearly directly overhead from the HobbyEberly Telescope, and Cen X-4, which is sometimes nearly directly overhead from the site of the SALT telescope. Boron abundance measurements would be similarly useful, but can be made only from space.

\section{Conclusions}

It is shown here that the tidal locking of the mass donors in $\mathrm{X}$-ray binaries should result in an enhancement in lithium abundance of a factor of about 5 when compared with what would be expected from single stars of the same age and mass. Tidal locking is therefore likely to be an important factor in producing the lithium overabundances seen in X-ray binaries' counterparts. It could be the sole factor in the event that the effects of tidal locking are more important in the tighter X-ray binaries than they are in the tidally locked binaries which have been observed in open clusters, or if the X-ray binaries are typically younger than a few Gyr.

\section{References}

Anders, E., \& Gravesse, N. 1989, GeCoA, 53, 197

Bailyn, C. D., \& Grindlay, J. E. 1990, ApJ, 353, 159

Bildsten, L., \& Rutledge, R. E. 2000, ApJ, 541, 908

Brocksopp, C., Bandyopadhyay, R. M., \& Fender, R. P. 2004, New Astron., 9, 249

Butt, Y., Maccarone, T. J., \& Prantzos, N. 2003, ApJ, 587, 748

Clark, G. W. 1975, ApJ, 199, L143

Fabian, A. C., Pringle, J. E., \& Rees, M. J. 1975, MNRAS, 172, 15

Fender, R. P. 2005, in Compact Stellar X-ray Sources, ed. W. H. G.

Lewin, \& M. van der Klis (Cambridge University Press), in press [arXiv:astro-ph/0303339]

Filippenko, A. V., Matheson, T., \& Barth, A. J. 1995a, ApJ, 455, L139

Garcia, M. R., Callanan, P. J., McCarthy, J., Eriksen, K., \& Hjellming, R. M. 1999, ApJ, 518, 422 
Guessoum, N., \& Kazanas, D. 1999, ApJ, 512, 332

Harlaftis, E. T., Horne, K., \& Filippenko, A. V. 1996, PASP, 1081, 762

Hills, J. G. 1976, MNRAS, 175, 1

King, A. R., Kolb, U., \& Burderi, L. 1996, ApJ, 464, L124

Marsh, T. R., Robinson, E. L., \& Wood, J. H. 1994, MNRAS, 266, 137

Martin, E. L., Rebolo, R., Casares, J., \& Charles, P. A. 1994, ApJ, 435, 791

Martin, E. L., Rebolo, R., Casares, J., \& Charles, P. A. 1995, A\&A, 303, 785

Martin, E. L., Casares, J., Molaro, P., Rebolo, R., \& Charles, P. A. 1996, New Astron., 1, 197

Meyer-Hofmeister, E. 2004, A\&A, 423, 321
Migliari, S., Fender, R. P., Rupen, M., et al. 2003, MNRAS, 342, L67

Pinsonnault, M., Kawaler, S. D., \& Demarque, P. 1990, ApJS, 74, 501

Pinsonneault, M. H., Kawaler, S. D., Sofia, S., \& Demarque, P. 1989, ApJ, 338, 424

Portegies Zwart, S. F., Dewi, J., \& Maccarone, T. 2004, MNRAS, 355, 413

Ryan, S. G., \& Deliyannis, C. P. 1998, ApJ, 500, 398

Sabbi, E., Gratton, R. G., Bragaglia, A., et al. 2003, A\&A, 412, 829

Soderblom, D. R., Oey, M. S., Johnson, D. R. H., \& Stone, R. P. S. 1990, AJ, 99, 595

van den Heuvel, E. P. J., \& Bitzaraki, O. 1995, A\&A, 297, L41

Yi, I., \& Narayan, R. 1997, ApJ, 486, 363

Zahn, J.-P. 1994, A\&A, 288, 829 